\title{
CHARACTERIZING THE DYNAMICS AND TENSIONS IN THE SOCIAL PRACTICES OF LOW-ACHIEVING STUDENTS IN A KNOWLEDGE-BUILDING ENVIRONMENT
}

\author{
Yuqin Yang \\ Central China Normal University, NO.152 Luoyu Road, Wuhan, Hubei, P. R. China, 430079
}

\begin{abstract}
The study used activity systems analysis to characterize the processes, dynamics, and tensions in the social practices developed by a class of academic low-achievers in a knowledge-building environment augmented by analytics-supported reflective assessment. A class of 37 Grade 9 low-achievers and an experienced teacher participated this study. Classroom data sources including classroom videos and observations, student artifacts, student interviews, and videos of reflective-assessment sessions were collected and analyzed. The findings indicated that staging tasks, frequent and opportunistic knowledge-building talks, the framing of discourse improvement as collective responsibility, and the acquisition of new skills and interests served as new tools mediating the students' activities. Our findings have important implications for the design of technology-rich environments as metacognitive tools to support low achievers.
\end{abstract}

\section{KEYWORDS}

Knowledge Building, Academic Low-Achieving Students, Activity System Analysis

\section{INTRODUCTION}

Understanding how low-achievers develop social practices by which they gradually generate creative ideas is critical for low-achievers' continuous development. This study used activity systems analysis (ASA), developed by (Yamagata-Lynch, 2010), to map the development of, and the dynamics and tensions in, the social practices in which a class of low-achievers collectively advance their ideas in a community context. This study was part of a program that examined the design, process, and effects of knowledge building environments augmented by analytics-supported reflective assessment on low-achievers. The study addressed the following research questions:

1) What is the nature of social practices from a cultural historical activity theory (CHAT) perspective?

2) What characterizes the dynamics of the social practices from a CHAT perspective, and how do the dynamics drive the development of the social practices?

\section{REFLECTIVE ASSESSMENT AND LOW-ACHIEVING STUDENTS}

In reflective assessment, students use their agency to reflect on a set of criteria/principles or learning goals. They produce their own feedback based on continuous assessment of the inquiry process and product, and improve their ongoing learning by attacking broader problems and continuously creating knowledge (Scardmalia, 2002; White \& Frederiksen, 1998). Reflective assessment is collaborative: not everyone in the community needs to be highly metacognitive for the process to be effective, and students can scaffold others' metacognitive development through modeling. 
Research in the learning sciences suggests that students of various achievement levels can participate in and benefit from reflective assessment. For example, White and Frederiksen (1998) found that reflective assessments carried out by middle-school students positively affected their performance in a scientific-inquiry test and a physics test, and that the process was particularly beneficial for low-achieving students. Toth et al. (2002) reported a similar intervention in which students were guided by a reflective assessment rubric during the inquiry, and found that reflective assessment typically had a positive effect on learning. Herrenkohl et al. (2011) found that the use of self- and peer-assessment tasks in an interactive online environment, accompanied by assessment criteria and useful tools such as software advisors, can scaffold students' conceptual understanding in science. However, there has been relatively little research into how we might assist low-achieving students to carry out this assessment as active agents to improve their collaborative inquiry.

This study characterized and mapped the developmental process of social practices created by low-achieving students in a knowledge-building environment augmented by reflective assessment. In this study, we provided students an assessment tool, the Knowledge Connections Analyzer (KCA) (Yang et al., 2016) to support reflective assessment. The KCA is a Web-based analytics tool designed to be used by secondary-school students to reflect on their online work in a Knowledge Forum. It queries the Knowledge Forum database to collect information on four intuitive questions related to knowledge building. For a full description, see Yang et al. (2016).

\section{METHODS}

\subsection{Research Context and Participants}

The study was conducted at a Band-3 school in Hong Kong, which is at the 10th percentile. Students at such schools tend to be weaker in Chinese and English, communication, and critical thinking than their counterparts in Band-1 and Band-2 schools, and have poorer motivation, self-identity, self-image, and metacognitive skills. The participants were $379^{\text {th }}$ graders from a class taking a visual-arts course. They studied the topic of "Art appreciation" over five months in weekly one-hour lessons. Their teacher had previously found the students to be more motivated by knowledge building, a promising student-centered approach, than by other teaching methods. The teacher had considerable experience of teaching the visual arts, had taken a postgraduate course on knowledge building, and had used knowledge building for approximately eight years.

\subsection{Pedagogical Design}

The teacher used a three-phase pedagogical process to familiarize the students with knowledge building, as described in detail by Yang et al. (2016): (1) Creating a collaborative culture and helping students to build skills in inquiry, collaboration, and metacognition, (2) Deepening problem-centered inquiry in the Knowledge Forum, an online learning environment that supported students' online discourse, and (3) Using analytics-supported reflective assessment to foster deep domain understanding and metacognitive skills.

\subsection{Data Sources and Analysis}

\subsubsection{Data Sources}

In this study, we collected the following interrelated sets of qualitative data:

Classroom observations. We conducted the observations while the participants were engaged in activities related to knowledge building. The data comprised photographs and field notes related to some of the lessons, and video recordings of 17 one-hour lessons.

Video recordings of reflective-assessment sessions. We video recorded the students' reflective-assessment activities while on KF, in class, and during after-class group sessions, including their interpretations of and reflections on the data and plans for their KF discourse. 
Interviews. We conducted semi-structured interviews to obtain information about the students' experiences of KCA. Some of the interviews were conducted individually, and others in groups of 2-3 students. Most of the interviews were conducted informally in the computer laboratory in which the students used the KCA during class, and each interview lasted for 20-30 minutes. The interview questions corresponded to the four questions in the KCA to obtain information about students' use of the KCA to support reflective assessment. The interviews were audio recorded.

\subsubsection{Data Analysis}

We used ASA to characterize the social practices that arose from the KCA-aided reflective assessment during knowledge building. ASA has been used in several studies to identify the systemic contradictions and tensions that shape developments in educational settings (Barab et al., 2002; Kim, 2011). The ASA was conducted in two main stages: identifying codes and analyzing activity systems.

Code identification. We began data analysis using the constant comparative method (Strauss \& Corbin, 1998). Analyses were conducted to explore the potential determinants of students' productive and unproductive use of the KCA to conduct reflective assessment, and to identify the elements and ongoing activities that supported the transformation of newly introduced artifacts into cultural tools. The multifaceted analysis results of students' online discourse (Yang et al., 2016) provided the basis for the qualitative ASA. The study began by examining video-recordings of reflective assessment sessions to identify productive and unproductive uses of the KCA. Next, the study analyzed the potential of reflective assessment to increase students' focus on the key learning goals of knowledge building. The study further systematically analyzed the remaining sources of data to cast light on the students' perceptions of the effectiveness of the KCA in supporting reflective assessment, their experiences of using the KCA to conduct reflective assessment, and the activities and other elements that assisted or hindered the students' productive use of the KCA. The goal of this analysis was to report on thematic findings on the participants' engagement in KCA-afforded reflective assessment and various activities and other factors that made their use of the KCA productive or unproductive.

Identifying activity systems. This task involved three main steps: (a) developing thick descriptions of the participants' experiences in narrative format, (b) conducting ASA of the narratives, and (c) identifying substantiated findings (Yamagata-Lynch, 2010). Before drafting narratives of the students' experiences of self-directed reflective assessment, we identified substantiated stories from the data. "Work done by the class" was viewed as an activity system subject to ASA, and Engeström's (1987) triangle was used to describe the component structure of each instance of this activity. Identifying activity systems was an iterative process that involved multiple stages of revision, during which the drafted narratives were also modified. After finalizing the narratives and the ASA, we began identifying substantiated findings.

Validity of ASA. Trustworthiness was enhanced through consistent observation, methodological triangulation, and a rigorous coding process. We observed the participants consistently for approximately five months. At the same time, we obtained necessary information about the students, the teacher, methods of instruction, and curriculum by observing the teacher's work in other classes, and drawing on our six-year research relationship with the teacher. We conducted methodological triangulation using multiple sources of data: observation, students' artifacts, interviews, and questionnaires.

\section{RESULTS}

\subsection{Results of ASA}

We constructed three activity systems to describe and explain the development of the reflective-assessment activities and the interaction of these activities (a) before the KCA student activity, (b) during the KCA student activity, and (c) immediately after the KCA student activity. The activity systems are shown in Figures 1-3.

Activity system A (Figure 1) comprised the students' knowledge-building activities before using the KCA to carry out reflective assessment. In this activity system, a variety of meditational tools enabled the students to advance their knowledge, develop skills in summarization, collaboration, and metacognitive reflection, and develop a collaborative knowledge building culture. For example, the construction of a 
knowledge-building wall helped low-achievers to make their ideas visible and public and created a collaborative culture conductive to subsequent productive knowledge building.

These tools motivated the students to invest much more effort in developing both face-to-face and online knowledge-building discourse, and to some extent improved their understanding of knowledge building as a collective enterprise focusing on idea improvement. However, the students still lacked motivation and showed weak inquiry, collaborative, and metacognitive skills, which led to tension (a): the need to attain an object despite lacking motivation and skills. In addition, the resource limitation that directed students' attention from individual note-writing to collective knowledge advancement, and urged students to continuously review, reflect on, and further synthesize/rise above community ideas, brought about tension (b): attaining the object with limited resources. Moreover, the traditional teacher-centered school culture and the associated norms for participation undermined the teacher's efforts to foster knowledge building and the students' efforts to carry out knowledge building, which brought about tension (c): the need to attain an object within a traditional teacher-centered school culture and the norms for participation in that culture. These tensions placed the student participants involved in activity system A in contradictory knowledge-building situations, and provided minimal support for their attempts to collaboratively advance their discourse. The forces created by these conditions militated against the subjects' efforts to attain the object.

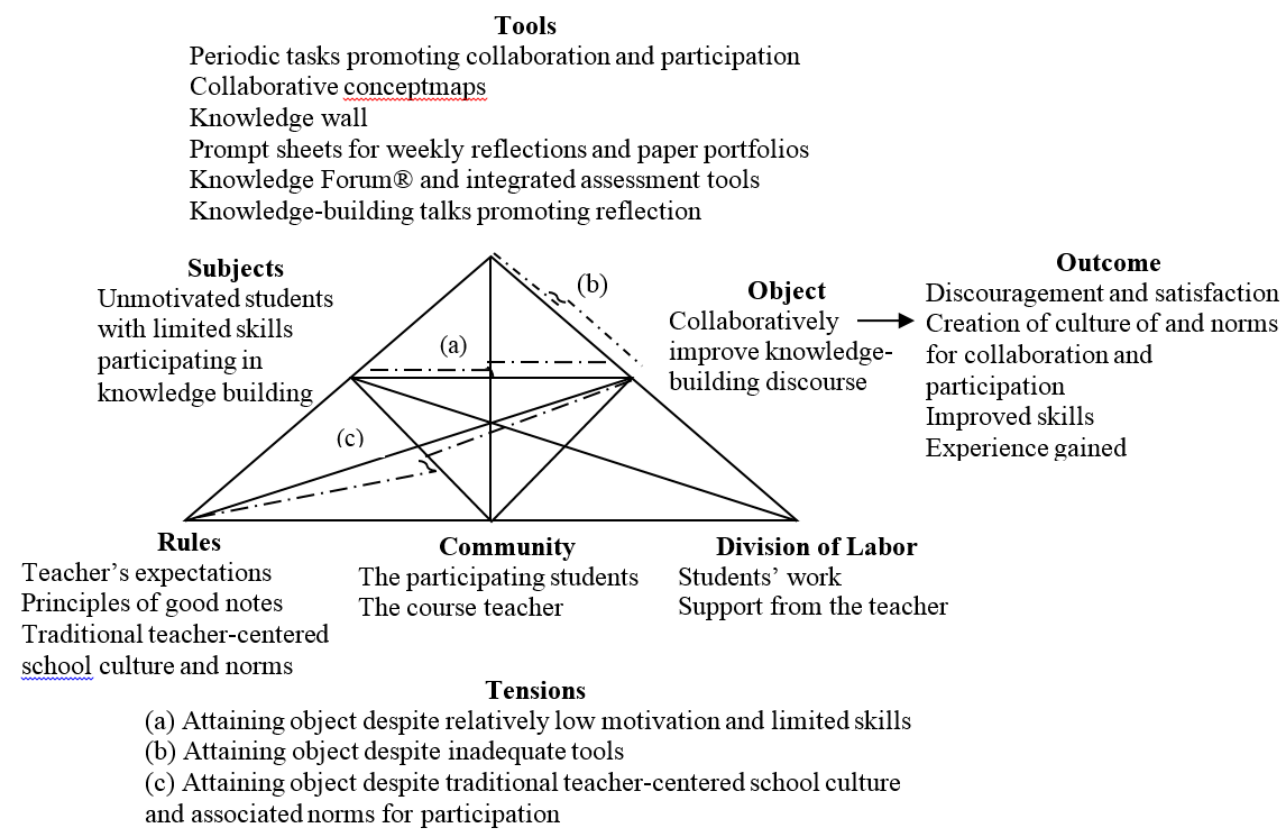

Figure 1. Activity System A: Before the KCA Student Activity

After the students engaged in reflective assessment using the KCA, the dynamics of the student activity systems changed: the students subsequently attempted to advance their knowledge-building discourse by carrying out KCA-based reflective assessment. Figure 2 shows the activity system at this stage (activity system B), a nested system incorporating the outcome of the earlier activity system (activity system A) (Yamagata-Lynch, 2010). In activity system B, the KCA was introduced to help the students engage in reflective assessment. They reflected on the KCA data and further synthesized and rose above the community's ideas. Initially, however, the students were unable to interpret the KCA data productively, leading to tension between the students' efforts to use the KCA data to improve their discourse and their ability to interpret the data. However, this tension lessened as the students gradually acquired the skills needed to interpret and use the KCA data and synthesize their ideas. Their skills development benefited from frequent and opportunistic knowledge-building talks promoting reflection on and thoughtful use of the KCA data, with an overarching emphasis on discourse improvement as a collective responsibility. For example, the following excerpt from the KCA reflection sheet demonstrates how the student was able to generate synthesis and rise above ideas collectively through reflective assessment with the KCA data, from the question "Are we putting our knowledge together?": 
We are wondering whether we have really put our knowledge together by referring to each other' notes, improved our understanding, and drawn conclusions...Look at the chart (pointing to the pie chart generated by the KCA), only $3 \%$ of our notes contain references... kind of very low... only $6 \%$ of our notes were used as references... You see, many of the summary notes are not quite good. Actually, we can further improve them (reading the pooled summary and rise-above note in the KCA)...we need to comment the notes referred to, for example why we choose them, why they are good or not...we need to refer more notes, summarize what they have talked about and identify further inquiry areas (from collective reflection in small groups)

In this excerpt, the students carried out KCA-supported reflective assessment with the purpose of inquiring ("whether we have really put our knowledge together by referring to each other' notes, improved our understanding, and drawn conclusions"), and analyze the possible issues ("only $3 \%$ of our notes contain references;" "kind of very low;" "only $6 \%$ of our notes were used as references;" "see, many of the summary notes are not quite good"). Based on their analysis, the group of students appeared to reflect on the quality of reference notes, made a plan ("further improve them") and took actions to address the issues ("we need to comment the notes referred to, for example why we choose them, why they are good or not...we need to refer more notes, summarize what they have talked about and identify further inquiry areas"). This example suggests that KCA-enhanced reflective assessment helped this student to connect their learning orientation to the knowledge-building goal of idea improvement as collective responsibility.

During this process, the students also became more motivated. As a result, tensions (a) and (b) in activity system A (the need to attain an object with limited skills and motivation, and the need to attain an object in the absence of a community-oriented framework for data-driven idea improvement) were substantially alleviated. In addition, during the KCA-driven reflective assessment, a range of tools (e.g., knowledge-building talks, a community-oriented framework for data-driven idea formation, collaborative concept maps, and strategies for developing collaboration skills and increasing motivation) helped the students to develop a knowledge-building culture. As a result, tension (c) in activity system A (the need to attain an object in the absence of a knowledge-building culture and associated norms for participation) was also substantially alleviated.

In activity system B, however, the teacher's expectations clashed with the students' limited attention, energy, and time. In addition, the expectations of the teacher and the research team clashed with students' attention, energy, and time. Tension (a) arose from the students' competing obligation to actualize the expectations of the teacher and research team and to complete their SBA projects. This tension is represented in a circular fashion because elements of the rule component created further tension in the system. Nevertheless, the overall outcome of activity system B was positive: the whole class collaboratively advanced their understanding by engaging in reflective assessment using KCA. Many of the students produced high-quality synthesis/rise-above notes, although others did not. The students continued to advance their collective understanding by gaining the skills, interests, and motivation necessary to pursue knowledge building. 


\section{Tools}

Increased motivation, skills, and experience and improved online discourse

$\mathrm{KCA}$ and corresponding prompt sheets

Knowledge Forum $\mathbb{B}$ and its integrated assessment tools

Collaborative conceptmaps

Knowledge-building talks promoting collaborative

reflection on and thoughtful use of the KCA data

Community-oriented framework for data-driven idea

improvement

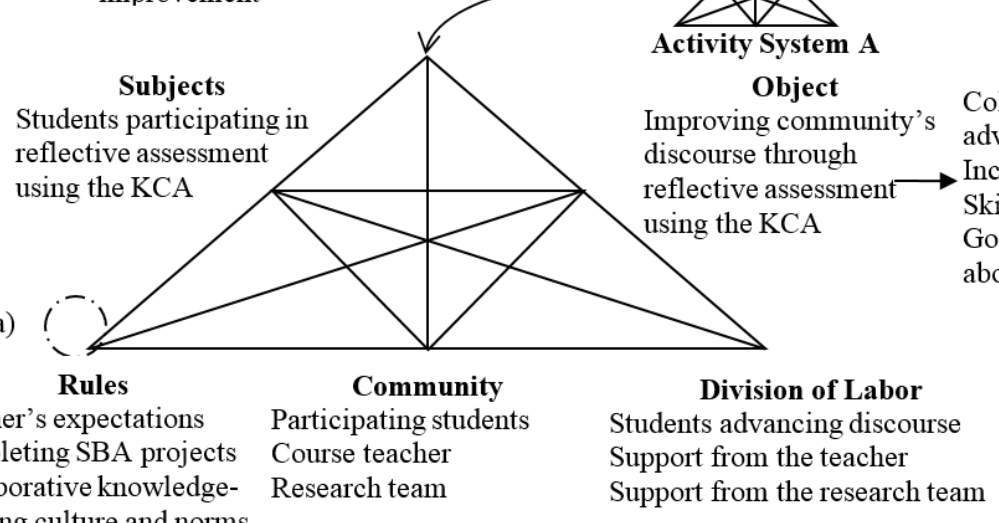

Tensions

(a) Actualizing the teacher's expectations while competing SBA projects

Figure 2. Activity System B: After the KCA student activity

Figure 3 shows Activity System $\mathrm{C}$ in which the knowledge-building participants are represented as subjects, and the intended collaborative improvement to the students' knowledge-building discourse is represented as the object. Capitalizing on the outcomes of activity system B, the students were able to collaboratively improve their knowledge-building discourse and develop skills in areas such as summarization, inquiry, collaboration, and metacognition. Many of the students also became interested in knowledge building, synthesizing notes, and analyzing their conceptual progress.

\section{Tools}

Knowledge Forum ${ }^{\circledR}$

Online discourse

Skills gained; increased motivation and interest

KCA data and accompanying prompt sheets



Collaborative knowledge-

building culture and norms

Figure 3. Activity System C: Immediately after the KCA Student Activity 


\subsection{Findings of the ASA}

The ASA yielded three main findings that explain the nature, dynamics and tensions of the social practices that arose from the students' KCA-afforded self-directed reflective assessment. These findings offer insights into the elements that became influential cultural tools, and the ongoing activities that supported the transformation of these newly introduced artifacts into cultural tools.

Regular tasks promoting inquiry, collaboration, and reflection introduced new tools that mediated student activities. Regular tasks were designed to help the students to develop skills in inquiry, collaboration, and reflection, and to create a collaborative culture and norms. Comparison of activity system A with activity system B revealed that these tasks constituted valuable tools mediating the students' activities. For example, the students exhibited limited cognitive skills (inquiry and summarization) and metacognitive skills (reflection) in activity system A, and seldom reflected on their existing (lack of) knowledge (based on informal individual student interviews at the beginning of the course). Therefore, the teacher encouraged the students to write weekly reflections and create individual paper portfolios with the help of a task sheet. These regular tasks enabled the students to gradually develop cognitive and metacognitive skills that mediated their subsequent activities. Writing paper portfolios cultivated the ability to summarize information, which in turn mediated the students' engagement in activity system B: some wrote synthesis notes in the style used to prepare the paper portfolios, i.e., identifying a string of good notes and explaining why these notes were good.

Framing discourse improvement as a collective responsibility acted as new tools that mediated new activities. As indicated in activity system A and activity system B, framing discourse improvement as a collective responsibility helped the students to understand over time that knowledge building requires a collective effort to improve ideas. A knowledge wall was used to frame discourse improvement as a collective responsibility before the students began working in the Knowledge Forum (see activity system A). After they were introduced to the KCA, the students were guided to reflect on the assessment data with an emphasis on data-driven discourse improvement as a collective responsibility. The students were encouraged to help each other to make sense of the data, engage in joint action planning, and discuss and share strategies for improving the community's discourse. The benefits of these activities became evident in activity systems $\mathrm{B}$ and $\mathrm{C}$, as the students collectively advanced the community's discourse by capitalizing on their collective reflections of the assessment data. Framing data-driven discourse improvement as a collective responsibility facilitated the creation of norms and fostered a sharing culture underpinned by a shared understanding of the processes and purposes of data use purposes and processes, and also stimulated student conversations about the data that improved the accuracy of their interpretations. Fostering norms and a culture that promotes critical inquiry, reflection, and the examination of underlying beliefs enables discourse to be improved through data use (Schunk \& Zimmerman, 1997).

New skills and increased interest were transformed into new tools that mediated the students' new activities. As indicated in activity system B and activity system C, the students used their newly gained skills and interests to engage in new activities that allowed them to progressively advance ideas in the communal space. For instance, in activity system B, the students used the collaboration skills learned in activity system A (through collaborative concept-mapping and collaborative knowledge-wall building) to engage in note writing in the Knowledge Forum and reflective assessment. The knowledge-building process required the students to collectively advance ideas and help each other to improve their notes. Combined with the skills gained in activity system A such as summarization, inquiry, collaboration, and metacognition, the students gradually exhibited increasing engagement in reflective assessment through the KCA and the production of knowledge-building discourse.

\section{CONCLUSION}

In this study, we found that various components of the activity systems shaped the students' knowledge-building behavior and reflective assessment in the KCA. These components were the skills required for data use, the establishment of a collaborative culture and norms for participation, the opportunities available for collaborative reflection about data, and the framing of data-driven discourse improvement in terms of collective responsibility. Our findings on the interactions between the conditions of 
and tensions within an activity system, and the processes by which such factors become either affordances or constraints to students' collaborative use of data, suggest ways of structuring change in classrooms and even schools, particularly to meet the needs of educationally disadvantaged students. Our findings also lay the groundwork for future research on students' collaborative work and metacognitive activities in relation to data use.

Our findings indicate that the KCA and its accompanying prompt sheets can help students to engage in productive reflective assessment and focus on the key learning goals of knowledge building, thereby collectively improving the discourse created by a community. Also, the findings suggest a design with three important components that are accessible and feasible for low-achievers: (a) establishing a collaborative culture and norms for participation, practicing cognitive skills such as summarization and concept mapping, (b) periodic tasks that promote collaborative reflection and thoughtful use of the KCA, and (c) framing the data-driven improvement of discourse as a collective responsibility (Scardmalia, 2002).

Within knowledge-building research, studies have mainly relied on online discourses and quantitative server-log data in the Knowledge Forum database. Very few studies have made use of qualitative data such as interview transcripts, student artifacts, and face-to-face teacher-student and student-student discourse to characterize the social practices that arise from knowledge building. However, to gain a fuller understanding of knowledge building, it is necessary to understand the relationship between online discourse and the nature and dynamics of the social practices that develop during knowledge building (Zhang et al., 2007). This study has addressed this gap in the literature.

\section{ACKNOWLEDGEMENT}

This research was partly supported by a grant to the author from Ministry of Education of the People's Republic of China (Grant No. 18YJC880107), and a grant to Drs Jan van Aalst and Carol Chan from the University Grants Committee (Grant No. 752508H). We thank the teacher and students for their participation.

\section{REFERENCES}

Barab, S. A. et al., 2002. Using activity theory to understand the systemic tensions characterizing a technology-rich introductory astronomy course. Mind, Culture, and Activity, Vol. 9, No. 2, pp. 76-107.

Herrenkohl, L. R. Et al., 2011. Pedagogical practices to support classroom cultures of scientific inquiry. Cognition and Instruction, Vol. 29, No. 1, pp. 1-44.

Kim, M. S., 2011. Toward activity-centered literacy: Teaching and learning Korean literacy in a multilingual Montreal context. Asia Pacific Education Review, Vol. 12, No. 3, pp. 447-461.

Scardmalia, M., 2002. Collective cognitive responsibility for the advancement of knowledge. In B. Smith (Ed.), Liberal education in a knowledge society (pp. 67-98). Chicago, IL: Open Court.

Schunk, D. H., and Zimmerman, B. J., 1997. Social origins of self-regulatory competence. Educational psychologist, Vol. 32, No. 4, pp. 195-208.

Strauss, A., and Corbin, J., 1998. Basics of qualitative research: Techniques and procedures for developing grounded theory (2nd ed.). Thousand Oaks, CA: Sage Publications.

Toth, E. E. et al., 2002. "Mapping to know": The effects of representational guidance and reflective assessment on scientific inquiry. Science Education, Vol. 86, No. 2, pp. 264-286.

White, B., and Frederiksen, J., 1998. Inquiry, modeling, and metacognition: Making science accessible to all students. Cognition and Instruction, Vol. 16, No. 1, pp. 3-118.

Yamagata-Lynch, L. C., 2010. Activity systems analysis methods: Understanding complex learning environments. New York, New York, N.Y.: Springer.

Yang, Y. et al., 2016. Fostering collective learning through knowledge building among students with low academic achievement. Proceedings of the 12th International Conference on Learning Sciences. Singapore, pp. 819-822

Yang, Y., et al, 2016. Reflective assessment in knowledge building by students with low academic achievement. International Journal of Computer-Supported Collaborative Learning, Vol. 11, No. 3, PP 281-311.

Zhang, J. et al., 2007. Socio-cognitive dynamics of knowledge building in the work of 9-and 10-year-olds. Educational Technology Research and Development, Vol. 55, No. 2, pp. 117-145. 